\title{
Survival and percolation probabilities in the field theory of growth models
}

\author{
Hans-Karl Janssen \\ Institut für Theoretische Physik III, Heinrich-Heine-Universität, \\ 40225 Düsseldorf, Germany \\ E-mail: janssen@thphy.uni-duesseldorf.de
}

\begin{abstract}
Survival and percolation probabilities are most important quantities in the theory and in the application of growth models with spreading. We construct field theoretical expressions for these probabilities which are feasible for perturbation expansions. The outstanding role of the absorbing noise is stressed to obtain survival probabilities monotonic decreasing with time. We briefly consider some fundamental growth models equipped with absorbing noise which are representations of known universality classes of spreading phenomena. The critical scaling properties of their survival and percolation probabilities are stated. In an appendix we consider shortly the renormalized field theory of compact directed percolation.

PACS numbers: 05.10.Gg, 05.40.-a, 05.45Df, 05.70.Jk, 64.60.Ak, 64.60.Ht
\end{abstract}

\section{Introduction}

The investigation of the formation and properties of random structures has been an exciting topic in statistical physics for many years (see e.g. [1, 2]). Of particular interest are random structures which are formed by local rules. These processes can often be expressed in the language of population growth. Stochastic processes of this type describe the essential features of a vast number of growth phenomena of populations near their extinction threshold, and are relevant to a wide range of models in physics, chemistry, biology, and sociology [3, 4, 5, 6, 7]. The transition between survival and extinction of such a growing population constitutes a nonequilibrium continuous phase transition phenomenon and is characterized by universal scaling laws. The extinct states without active individuals (interacting particles) are stochastically absorbing, i.e. in a finite system with a finite number of degrees of freedom an extinct inactive state is reached with probability one at a finite time. Beyond the critical transition point, where the replication rate is greater than a critical value, the systems are in the active state. The corresponding mean extinction time is practically infinite in systems with a great number of degrees of freedom. To find active steady states it is therefore allowed to take the infinite volume limit, the so-called thermodynamic limit, before the infinite time limit is done. Then, the probability $P_{\infty}$ that an active state, created by a localized 
seed is still alive after an infinite time is greater than zero beyond the critical point. Hence, this percolation probability serves besides the density of the active particles in the steady state, $\rho_{s t}$, as an order parameter for growth processes. On the other hand, in the absorbing phase, the phase below the critical replication rate, each active configuration dies after a finite time. Thus $P_{\infty}$ is zero here and the steady states are inactive. However, an interesting observable in both phases is the survival probability $P(t)$. It is the probability that a process started from a localized seed, e.g. an active particle at the origin of space and time, is still active at time $t$. Of course, we have generally $P(t) \rightarrow P_{\infty}$ for $t \rightarrow \infty$.

Near a critical point one is typically interested in the asymptotic universal longwavelength, long-time behavior of the observables. Besides the existing vast number of simulation methods and results (see e.g. [3]), a variety of analytical tools were developed for growth processes in the past decades. The method which introduces the continuum limit from the beginning and exploits the renormalization group to obtain universal properties is the renormalized stochastic field theory [8, 9, 10, 11]. At the heart of this method is the calculation of time dependent mean values, correlation and response functions of the observables by using path integrals. Basic ingredient is a generalized probability measure $\exp (-\mathcal{J})$, where the dynamic functional $\mathcal{J}$ (nowadays also called a response functional) is a functional of time and space dependent fields. The restriction of $\mathcal{J}$ to the only relevant terms in an expansion of the slowly varying fields defines a renormalizable field theory. This theory represents then a full universality class of systems with common asymptotic properties.

Although the field theory of growth processes are based on a probability measure, the direct calculation of the survival probability $P(t)$ and the percolation probability $P_{\infty}$ for growth processes by field theoretic means was not well developed in the past. There exists an attempt of Muñoz, Grinstein, and Tu 12] (henceforth cited as MGT) to devise a practical method to determine correctly the scaling properties of the survival probability. However, these authors erroneously assert: "In field theories with continuous variables ... the absorbing state is a set of measure zero in phase space and so can never actually be reached in finite time. Thus $P(t)$ is strictly equal to unity for all $t$, so the concept of the survival probability has no utility.", an argument that resembles Zenon's paradox on Achilles and the tortoise [13. They introduce therefore another quantity as a substitute for $P(t)$ with the correct scaling properties. But their argumentation seems to rest solely on a naive interpretation of the Langevin equation as an ordinary differential equation for a continuously differentiable function, and of a stationary solution of the corresponding Fokker-Planck equation for the probability density of the stochastic process $\phi$ in zero spatial dimension. However, this stationary solution has a non-integrable singularity at $\phi=0$, and is therefore not normalizable. Hence, it cannot represent any features of the correct probability density near the absorbing state $\phi=0$. MGT, without considering any boundary condition of the time dependent solution of the Fokker-Planck equation, conclude: "An arbitrary initial probability distribution therefore evolves in time toward a distribution weighted at values $\phi$ lying progressively closer to zero. Note, however, that 
$\phi$ cannot actually achieve the value 0 in finite time." However, to reveal such properties of the time dependent probability distribution one has to ascertain analytically the behavior of the solutions of the Fokker-Planck equation near and at the absorptive state. But this was not done by MGT. Hence, their arguments are far of being convincing. We will show that the validity of their conclusion depends heavily on the form of the underlying noise. For a noise called absorptive in the following, the absorbing state has a $\delta$-probability measure in the continuous phase space. This measure accumulates more and more mass during the course of time. Consequently, the survival probability $P(t)$ is a monotonically decreasing function also in a continuous phase space. In contrast, for the so-called multiplicative noise [14] the assertions MGT are correct, and $P(t)=1$ for all finite times.

In this paper we develop expressions for survival and percolation probabilities of several reaction-diffusion processes with absorbing states in non-zero spatial dimensions from which the calculation of these quantities by field-theoretical methods can be performed. We show the feasibility of our expressions for the calculation of nontrivial probabilities in a mean field (saddle point) approximation that becomes correct for spatial extended systems above their higher critical dimension.

The outline of our paper is as follows. In section II we present a simple toy model that shows the fundamental difference between absorptive and multiplicative noise. It provides a nontrivial survival probability in the absorbing case. Section III reviews well known field theoretic results for directed percolation (DP) and its properties under the duality transformation. In section IV we derive the field theoretic expressions of the survival and the percolation probability for DP. Asymptotic scaling forms are found that are related to the mean particle density of the dual process. In section $\mathrm{V}$ we calculate the probabilities in a saddle point approximation. In section VI we study a variant of DP equipped with multiplicative noise. We show that in this case the survival probability is equal to one for all finite times. We compare in this section our result for the survival probability with the expression introduced by MGT. In Sec. VII we apply our results to the field theories of various fundamental growth processes. A short epilogue is given in section VIII. An appendix contains a brief presentation of the renormalized field theory of compact directed percolation.

\section{A toy model}

For the ease of the reader we re-consider in this section a well known example of a continuous growth process for which survival probabilities can be easily discussed in a concise way. Most of this material can be found in textbooks [15, 16, 17, 18, but it does not seem much appreciated by those working in this research field. The stochastic version of the logistic or Malthus-Pearl-Verhulst equation, $\dot{x}=-r(x) x+\zeta$ with $r(x)=(\tau+g x)$, describes the growth of a population size $x(t) \geq 0$. Equipped with absorptive noise $\zeta$, it provides a zero-dimensional toy-model of directed percolation. The model is also useful for the mean field description of the evolution of a homogeneously distributed population 
in a spatial extended system with volume $V$. In this case one defines the particle density by $n(t)=x(t) / V$. The coupling constant scales with the volume as $g \sim 1 / V$. The Gaussian random noise $\zeta(t)$ is characterized by correlations $\overline{\zeta(t) \zeta\left(t^{\prime}\right)}=2 x(t)^{\alpha} \delta\left(t-t^{\prime}\right)$. For physical growth processes these correlations as functions of $x, t$, and $t^{\prime}$ result from the elimination of many fast microscopic degrees of freedom in local equilibrium with the population size $x$. Therefore, they should be analytic functions in $x$ and zero if $x=0$. Hence, in the limit where microscopic correlation times go to zero on the slow macroscopic time-scale, we typically find for population processes $\alpha=1$ (absorptive noise) or $\alpha=2$ (multiplicative noise) in a small $x$ expansion neglecting higher orders. The nowadays popular terminology "multiplicative noise" stems from the fact that in the before-mentioned time limit, the noise may be formally written as a product of an analytic function of $x$ and of white noise. In any event, it is essential that the noise is interpreted in the Itō-sense [17, 18. A very qualitative glance on the logistic Langevinequation shows that the random impulses resulting from the noise scale like $x^{\alpha / 2}$. Thus, for small $x$, these impulses can easily surmount the distance $x$ to the absorbing state $x=0$ if $\alpha<2$. We expect that this is the condition for which a non-zero extinction probability $P_{0}(t)=1-P(t)$ for finite times can be defined.

Nevertheless, a more satisfactory tool for the analytical description of this stochastic process is given by the corresponding Fokker-Planck equation for the probability density $p(x, t)$ to find a population with $x \geq 0$ at time $t$ :

$$
\frac{\partial p(x, t)}{\partial t}=\frac{\partial[r(x) x p(x, t)]}{\partial x}+\frac{\partial^{2}\left[x^{\alpha} p(x, t)\right]}{\partial x^{2}} .
$$

The Fokker-Planck equation is also known as the forward Kolmogorov equation for the fundamental probability density $p(x, t)=w\left(x, t \mid x^{\prime}, t^{\prime}\right)$ with $t \geq t^{\prime}$ and the initial condition $w\left(x, t^{\prime} \mid x^{\prime}, t^{\prime}\right)=\delta\left(x-x^{\prime}\right)$ with $0<x^{\prime}<\infty$. As a second order partial differential equation it needs in general two boundary conditions at $x=0$ and $x=\infty$. Starting in the open interval $(0, \infty)$, it may be that the stochastic process $x(t)$ does never takes on the value $x=0$ or $x=\infty$ in finite times. In this case the corresponding boundary is called natural, and no boundary condition at this value has to be imposed. In contrast, if the process $x(t)$ has positive probability of taking the value $x=0$ or $x=\infty$, the corresponding boundary is called accessible. For accessible boundaries there are two possible cases:

(i) Exit: the drift towards the boundary is such that the boundary automatically acts as an absorbing barrier, and no boundary condition can be imposed.

(ii) Regular: the process behaves like a classical diffusion process near the boundary, and various boundary conditions can be imposed.

Hence, for the existence of an unbiased nontrivial extinction probability, the absorbing state at $x=0$ should be an exit boundary.

According to the scheme of Feller [15], the classification of boundaries depends on 
the Lebesgue integrability of the function

$$
\begin{aligned}
F(x) & =\exp \left\{-\int_{x_{0}}^{x} \mathrm{~d} y r(y) y^{1-\alpha}\right\} \\
& =\exp \left\{\frac{\tau\left(x_{0}^{2-\alpha}-x^{2-\alpha}\right)}{2-\alpha}+\frac{g\left(x_{0}^{3-\alpha}-x^{3-\alpha}\right)}{3-\alpha}\right\},
\end{aligned}
$$

where $x_{0} \in(0, \infty)$. The following functions are defined besides $F(x)$ :

$$
\begin{aligned}
& G(x)=\frac{F(x)}{x^{\alpha}}, \\
& H(x)=F(x)^{-1} \int_{x_{0}}^{x} \mathrm{~d} y G(y) .
\end{aligned}
$$

Note that the stationary solution of the Fokker-Planck equation (2.1), $G(x)$, is a stationary probability distribution only if it is normalizable. We denote with $f(x) \in \mathcal{L}(0)$ or $f(x) \in \mathcal{L}(\infty)$ a function that is Lebesgue integrable on the interval $\left(0, x_{0}\right)$ or $\left(x_{0}, \infty\right)$, respectively. Then Feller's classification criteria are:

(i) The boundary $b$ is regular if $F(x)^{-1} \in \mathcal{L}(b), G(x) \in \mathcal{L}(b)$, and $H(x) \in \mathcal{L}(b)$;

(ii) The boundary $b$ is exit if $F(x)^{-1} \in \mathcal{L}(b), G(x) \notin \mathcal{L}(b)$ and $H(x) \in \mathcal{L}(b)$;

(iii) The boundary $b$ is natural in the other cases.

Applying these criteria one finds easily that for $\alpha<3$ the boundary at infinity is natural, whereas the boundary at zero is regular for $\alpha<1$, exit for $1 \leq \alpha<2$, and natural for $2 \leq \alpha$ (attractive for $\tau>-1$, repulsive for $\tau \leq-1$ ). It is now easily seen that for $1 \leq \alpha<2$ the Fokker-Planck equation (2.1) is transformed back to the case $\alpha=1$ by the setting $y=x^{2-\alpha}$ and the replacement $r(x) \rightarrow \tilde{r}(y)=r(x)+(\alpha-1) /(2-\alpha)$. Hence, in the following we concentrate on the absorptive noise $\alpha=1$ and to the multiplicative noise $\alpha=2$. In both cases no additional boundary conditions are needed for the solution of the initial problem.

On the other hand we find from above that there is a fundamental difference between these two cases: for absorptive noise there is a non-zero probability for the stochastic process to reach the absorbing state at $x=0$ in finite times, whereas for multiplicative noise the probability is zero that the process reaches $x=0$ in finite times. This different behavior is reflected in the evolution of the probability distribution in time: for absorptive noise the probability density shows a $\delta$-peak directly at the absorbing boundary which accumulates more and more mass in the course of time. For multiplicative noise in the case $\tau>-1$ the probability distribution $p(x, t)$ represents a peak, which moves to the left, becomes smaller and smaller in extension, and accumulates all probability near the absorbing state $x=0$ without reaching this state itself $(p(0, t)=0$ for $t<\infty)$. Only in the infinite time limit we get $\lim _{t \rightarrow \infty} p(x, t)=\delta(x)$ and the stochastic process is ultimately absorbed. For $\tau<-1$ the probability distribution with $p(0, t)=0$ for $t<\infty$ converges to the stationary distribution $\lim _{t \rightarrow \infty} p(x, t) \propto G(x)$, equation (2.3a). However, note that the limits $\lim _{t \rightarrow \infty}$ and $\lim _{x \rightarrow 0}$ cannot be interchanged [20]. 
The fundamentally different behavior of the distributions in time can be made fully explicit in the linear case $r(x)=\tau$ [16, 18, 20]. If the noise is absorptive, $\alpha=1$, the solution of the Fokker-Planck equation (2.1) is found easily by a Laplace transform to

$$
\begin{aligned}
& p\left(x, t \mid x_{0}\right)=\delta(x) P_{0}\left(t, x_{0}\right)+\theta(x) \bar{p}\left(x, t \mid x_{0}\right), \\
& \bar{p}\left(x, t \mid x_{0}\right)=h(t) \sqrt{\bar{x}(t) / x} I_{1}(2 h(t) \sqrt{\bar{x}(t) x}) \exp [-h(t)(\bar{x}(t)+x)], \\
& P_{0}\left(t, x_{0}\right)=\exp [-h(t) \bar{x}(t)] .
\end{aligned}
$$

$I_{1}$ is the modified Bessel function of first order, and we have defined

$$
\begin{aligned}
& \bar{x}(t)=x_{0} \exp (-\tau t), \\
& h(t)=\frac{\tau}{1-\exp (-\tau t)} .
\end{aligned}
$$

Note that the extinction probability $(2.4 \mathrm{C})$ increases in the course of time monotonically from 0 to 1 (or $\exp \left(-|\tau| x_{0}\right)$ if $\tau<0$, whereby $P_{\infty}\left(x_{0}\right)=\lim _{t \rightarrow \infty}\left(1-P_{0}\left(t, x_{0}\right)\right)=$ $1-\exp \left(-|\tau| x_{0}\right)$ is a kind of percolation probability). Hence, it is explicitly shown that the assertion "the stochastic process cannot actually achieve the value 0 in finite time" of MGT is wrong in the case of absorptive noise.

The behavior of the probability distribution is qualitatively different in the case of multiplicative noise, $\alpha=2$. Then, the Fokker-Planck equation (2.1) is solved by the substitution $x=\exp y$ that transforms the stochastic process to simple diffusion with drift. One obtains

$$
p\left(x, t \mid x_{0}\right)=(\sqrt{4 \pi t} x)^{-1} \exp \left(-\left[\ln x / x_{0}+(\tau+1) t\right]^{2} / 4 t\right) .
$$

Indeed, here $p\left(0, t \mid x_{0}\right)=0$ and $P_{0}\left(t, x_{0}\right)=0$ for all finite times, and the assertion of MGT is correct.

The fundamental probability density $w\left(x, t \mid x^{\prime}, t^{\prime}\right)$ must also be a solution of the backward Kolmogorov equation

$$
\frac{\partial w\left(x, t \mid x^{\prime}, t^{\prime}\right)}{\partial t^{\prime}}=r\left(x^{\prime}\right) x^{\prime} \frac{\partial w\left(x, t \mid x^{\prime}, t^{\prime}\right)}{\partial x^{\prime}}-x^{\prime \alpha} \frac{\partial^{2} w\left(x, t \mid x^{\prime}, t^{\prime}\right)}{\partial x^{\prime 2}} .
$$

With the Ansatz $w\left(x, t \mid x^{\prime}, t^{\prime}\right)=\delta(x) P_{0}\left(t-t^{\prime}, x^{\prime}\right)+\theta(x) \bar{p}\left(x, t \mid x^{\prime}, t^{\prime}\right)$ one formally extends the range of the variable $x$ in (2.1) to the open interval $(-\infty, \infty)$. Then $\int_{-\infty}^{\infty} \mathrm{d} x p\left(x, t \mid x^{\prime}, t^{\prime}\right)=P_{0}\left(t-t^{\prime}, x^{\prime}\right)+\int_{0}^{\infty} \mathrm{d} x \bar{p}\left(x, t \mid x^{\prime}, t^{\prime}\right)=1$ where $\bar{p}\left(x, t \mid x^{\prime}, t^{\prime}\right)$ fulfills the original Kolmogorov equations. For $P_{0}\left(t, x^{\prime}\right)$ one gets the ordinary differential equation

$$
\dot{P}_{0}\left(t-t^{\prime}, x^{\prime}\right)=-\frac{d}{d t} \int_{0}^{\infty} \mathrm{d} x \bar{p}\left(x, t \mid x^{\prime}, t^{\prime}\right)=\alpha \lim _{x \rightarrow 0}\left(x^{\alpha-1} \bar{p}\left(x, t \mid x^{\prime}, t^{\prime}\right)\right) .
$$

$\dot{P}_{0}$ is zero for multiplicative noise but equal to $\bar{p}\left(0, t \mid x^{\prime}, t^{\prime}\right)$ for absorptive noise. From the backward equation (2.6) on derives the differential equation of the survival probability $P\left(t, x^{\prime}\right)=1-P_{0}\left(t \mid x^{\prime}, t^{\prime}=0\right)$ starting from a state with population size $x^{\prime}>0$ at $t^{\prime}=0$. Using the time translation invariance of the process, the time evolution follows from the backward Kolmogorov equation (2.6) as

$$
\frac{\partial P(t, x)}{\partial t}=-r(x) x \frac{\partial P(t, x)}{\partial x}+x^{\alpha} \frac{\partial^{2} P(t, x)}{\partial x^{2}}
$$


with $P(0, x)=1$ for $x>0$. Using this equation the mean time $T(x)=\int_{0}^{\infty} \mathrm{d} t P(x, t)$ to reach the absorbing boundary at $x=0$ from a state $x>0$ is easily calculated

$$
T(x)=\int_{0}^{x} \mathrm{~d} y \int_{0}^{\infty} \frac{\mathrm{d} s}{(s+y)^{\alpha}} \exp \left(-\int_{y}^{s+y} \mathrm{~d} z z^{1-\alpha} r(z)\right) .
$$

With $r(x)=\tau+g x$ one obtains in the case of multiplicative noise

$$
T(x)=\int_{0}^{x} \mathrm{~d} y y^{\tau} \int_{0}^{\infty} \frac{\mathrm{d} s}{(s+y)^{2+\tau}} \exp (-g s) .
$$

This expression diverges at the lower integration boundary, $y=0$, irrespectively of the sign of $\tau$ for all $x>0$. Hence, as we already know, the stochastic process cannot reach the absorbing state in finite time.

In the case of absorptive noise we obtain

$$
T(x)=\int_{0}^{x} \mathrm{~d} y \int_{0}^{\infty} \frac{\mathrm{d} s}{s+y} \exp \left[-g s^{2} / 2-(\tau+g y) s\right] .
$$

This expression is finite for all $x>0$ and shows again that the extinction probability to reach the absorbing state is nonzero even for finite times. From the mean field point of view, starting with a homogeneous state in the volume $V$, we have $x \sim V$ and $g \sim 1 / V$. Consequently, for $\tau>0$ the mean extinction time scales with the volume as $T(x) \sim \tau^{-1}|\ln x|$, whereas for $\tau<0$ we have an exponential increase $\ln T(x) \sim \tau^{2} / g$. This profound difference between the absorbing and the active phase of population growth is also expected in extended but finite systems.

Our discussion of known results from the analysis of continuous stochastic processes has shown that the behavior of the survival probability in time depends crucially on the type of the noise. It is shown that there exists a non-trivial survival probability in a theory with absorptive noise also in a phase space of a continuous variable. Thus, the toy model does not provide any argument against the existence of a non-trivial survival probability in the case of field theories of growth processes in spatial dimensions greater than zero. Indeed, in the next chapters we directly construct calculable expressions of survival and percolation probabilities for such field theories in terms of path integrals.

\section{Directed percolation}

In order to be consistent with later parts of the article, we will concisely present well known material on critical stochastic processes with absorbing states in this section. We will concentrate on directed percolation (DP) as the typical growth model [21, 22, 23, 24, 25, 28, 26]. For an comprehensive overview see [3, 11].

From the onset we use a mesoscopic picture in which all microscopic length- and time-scales are considered to be very short. We are interested in the asymptotic properties of the growth process near the critical point between the absorbing phase and the steady active phase. Thus, we take a continuum approach with the density of the active particles, $n(\mathbf{x}, t)$, as the stochastic variables. We remove all irrelevant contributions to the equations of motion. The growth process might be represented by 
a Langevin equation (in the Itō sense), which is constructed in accordance with the principle of the existence of an absorbing state as [25, 27, 28,

$$
\partial_{t} n=\lambda \nabla^{2} n-R[n] n+Q+\zeta,
$$

where $\zeta(\mathbf{x}, t)$ denotes the noise which has to vanish if $n(\mathbf{x}, t)=0$. The reaction rate $R[n]$ models birth and death of particles, as well as the saturation of the population. In addition we introduce an external particle source $Q(\mathbf{x}, t)$. The Langevin source $\zeta(\mathbf{x}, t)$ can be assumed to be Gaussian with absorbing correlation

$$
\overline{\zeta(\mathbf{x}, t) \zeta\left(\mathbf{x}^{\prime}, t^{\prime}\right)}=\lambda g^{\prime \prime} n(\mathbf{x}, t) \delta\left(\mathbf{x}-\mathbf{x}^{\prime}\right) \delta\left(t-t^{\prime}\right) .
$$

Diffusive noise is irrelevant in comparison to the reaction noise, equation (3.2), in the case of DP, but in general indispensable for processes with multiplicative noise [25, 29]. The extinction rate is given by

$$
R[n]=\lambda\left(\tau+\frac{g^{\prime}}{2} n\right) .
$$

Under the influence of fluctuations, the critical point is found at a value $\tau=\tau_{c}<0$. We implicitly renormalize this parameter by $\tau \rightarrow \tau-\tau_{c}$ so that the critical point is always found at $\tau=0$.

The field $Q(\mathbf{x}, t) \geq 0$ of the Langevin equation (3.1) describes the external source distribution of particles. As a special case the creation of one particle as a seed at the origin $\mathbf{x}=0$ and $t=0$ is represented by a source term

$$
Q(\mathbf{x}, t)=\delta(\mathbf{x}) \delta(t) .
$$

On the other hand, the choice $Q=\lambda h=$ const describes the creation of particles at a constant rate $Q$ uniform in space and time. In the following we are interested in the particular source model

$$
Q(\mathbf{x}, t)=\lambda h+\rho_{0} \delta(t)=\lambda\left(h+\rho_{0} \delta(\lambda t)\right),
$$

where $\rho_{0}$ constitutes a homogeneous particle density as an initial condition.

Dynamic response functionals $\mathcal{J}$ [8, 9, 10] based on Langevin equations of the form (3.1) with noise correlation (3.2) have the general form [27, 25, 28]

$$
\mathcal{J}[\tilde{n}, n]=\int \mathrm{d} t \mathrm{~d}^{d} x\left\{\lambda \tilde{n}\left(\lambda^{-1} \frac{\partial}{\partial t}+\left(\tau-\nabla^{2}\right)+\mathcal{V}[\tilde{n}, n]\right) n\right\} .
$$

Here, $\tilde{n}(\mathbf{x}, t)$ denotes the response field conjugated to the particle density $n(\mathbf{x}, t)$. For DP we have

$$
\mathcal{V}[\tilde{n}, n]=\frac{1}{2}\left(g^{\prime} n-g^{\prime \prime} \tilde{n}\right)
$$

The introduction of the source $Q$ leads to the shift

$$
\mathcal{J}[\tilde{n}, n] \rightarrow \mathcal{J}_{Q}[\tilde{n}, n]=\mathcal{J}[\tilde{n}, n]-(Q, \tilde{n})
$$

where $(Q, \tilde{n})$ denotes the integral $\int \mathrm{d} t \mathrm{~d}^{d} x Q(\mathbf{x}, t) \tilde{n}(\mathbf{x}, t)$. The responses are defined with respect to the particle source $Q(\mathbf{x}, t) \geq 0$. They are represented by correlation functions with the response field $\tilde{n}(\mathbf{x}, t)$. Such response and correlation functions (called Green's 
functions in the following) can now be calculated by path integrals with the probability weight $\exp (-\mathcal{J})$. We use the notation

$$
\langle\mathcal{A}[n, \tilde{n}]\rangle=\int \mathcal{D}(\tilde{n}, n) \mathcal{A}[n, \tilde{n}] \exp (-\mathcal{J}[\tilde{n}, n]),
$$

where $\mathcal{A}[n, \tilde{n}]$ is any functional of the fields $n, \tilde{n}$, and $\mathcal{D}(\tilde{n}, n)$ denotes the integral measure which is properly normalized $\langle 1\rangle=1$. In a suitable discretization it reads $\mathcal{D}(\tilde{n}, n)=\prod_{\mathbf{x}, t}(d \tilde{n}(\mathbf{x}, t) d n(\mathbf{x}, t) / 2 \pi i)$. For practical calculations, the observables $\mathcal{A}[n, \tilde{n}]$ should be either polynomial or exponential. Then a diagrammatic perturbation theory can be developed. The integration of the response fields is done along the imaginary axis, but it can be deformed to general complex values in finite regions. The integration of the density field can be formally extended over the full real axis. Initial and final conditions are supplied by

$$
n(\mathbf{x},-\infty)=\tilde{n}(\mathbf{x}, \infty)=0
$$

for all $\mathbf{x}$. For $Q \equiv 0$ we have therefore

$$
\langle n(\mathbf{x}, t)\rangle=\langle\tilde{n}(\mathbf{x}, t)\rangle=0
$$

for all $\mathbf{x}$ and $t$.

Now we define the duality transformation (known as rapidity reversal in Reggeon field theory and corresponding to duality in the mathematical theory of interacting particle systems [6]) as

$$
\alpha^{-1} \tilde{n}(\mathbf{x}, t)=: \tilde{s}(\mathbf{x}, t) \longleftrightarrow-s(\mathbf{x},-t):=-\alpha n(\mathbf{x},-t) .
$$

The free, bilinear, part of the dynamic functional (3.6) is invariant under this transformation for each finite $\alpha$. Note that $\alpha^{2}$ has the dimension of a spatial volume. Choosing $\alpha=\sqrt{g^{\prime} / g^{\prime \prime}}$ the interaction part of DP, $\mathcal{V}[\tilde{n}, n]$ (3.7), is (up to the time inversion) also invariant under duality. Of course, $\alpha$ is a redundant variable from the renormalization group point of view. Such variables should be eliminated before any renormalization group consideration is applied. Choosing therefore $s$ and $\tilde{s}$ as the fundamental fields, the invariant coupling is $g=\sqrt{g^{\prime} g^{\prime \prime}}$, and the fields $s$ and $\tilde{s}$ have the same scaling dimensions in DP. For general growth models, the duality transformation defines a dual stochastic process which can be different from the original one. Such a growth model is therefore not self-dual like DP. The quantities belonging to the dual process are designed in the following by a hat (e.g. the mean particle density of the dual process is denoted by $\hat{\rho}$ ).

As a last point we note the asymptotic scaling properties of the Green's functions, the cumulants of the variables $n$ and $\tilde{n}$. One obtains

$$
\begin{aligned}
& \left\langle\prod_{i=1}^{N} n\left(\mathbf{x}_{i}, t_{i}\right) \prod_{j=1}^{\tilde{N}} \tilde{n}\left(\mathbf{x}_{j}, t_{j}\right)\right\rangle^{(\mathrm{cum})}=|\tau|^{N \beta+\tilde{N} \tilde{\beta}} \\
& \quad \times F_{N, \tilde{N}}\left(\left\{|\tau|^{\nu} \mathbf{x},|\tau|^{\nu z} t\right\}, h /|\tau|^{(d+z) \nu-\tilde{\beta}}, \rho_{0} /|\tau|^{d \nu-\tilde{\beta}}\right)
\end{aligned}
$$

with universal functions $F_{N, \tilde{N}}$. Particular scaling laws follow from this asymptotic result of the renormalization group equation. It shows that in general the scaling of growth 
processes is defined by four independent scaling exponents $z, \nu, \beta, \tilde{\beta}$ [30]. However, for theories which are self-dual like DP we have in addition the relation $\beta=\tilde{\beta}$.

\section{Percolation and survival probabilities for directed percolation}

The expressions for the percolation and the survival probability for DP which are constructed in this section, can also be used, mutatis mutandis, for other growth processes, briefly considered in a later section.

Percolative spreading processes with absorbing states like DP provide two independent fundamental order parameters, the density of the active particles in the steady state $\rho_{s t}$, and the percolation probability $P_{\infty}$. Near the transition point they scale as $\rho_{s t} \sim|\tau|^{\beta}$ and $P_{\infty} \sim|\tau|^{\beta^{\prime}}$ where, in general, the two exponents $\beta$ and $\beta^{\prime}$ are distinct from each other [30]. It is well known that this asymptotic scaling behavior follows from the relations $\rho_{s t} \sim\langle n\rangle$ and $P_{\infty} \sim\langle\tilde{n}\rangle$, where, in particular, one has to specify the meaning of the expectation value for the latter expectation value. Due to the duality transformation (3.12), $P_{\infty}$ is intimately related to the particle density of the dual process: $P_{\infty} \sim \hat{\rho}_{s t}$, so that $\beta^{\prime}=\tilde{\beta}=\hat{\beta}$. Hence, we get for self dual processes (like DP) the well known relation $\beta^{\prime}=\beta$. In order to calculate scaling functions and universal amplitude ratios it is, however, not sufficient to consider only the asymptotic scaling behavior. We have to derive the exact relation between the two order parameters.

The discussion of the cluster probabilities is complicated by the fact that one has to deal carefully with the probabilities coming from the non-scaling small clusters, say, with particle numbers smaller than $N_{0}$. For these small clusters the asymptotic continuum field theory cannot be used. However, the small clusters do not induce critical singularities due to their finiteness. We assume as usual that they provide only analytical corrections [1].

Let $p_{N}(\tau)$ be the probability for a directed cluster in $(d+1)$-dimensional spacetime consisting of $N$ particles created from one particle, the seed, at the origin of the

space-time lattice, which is described by the source (3.4). For $N>N_{0} \gg 1$ one can use the continuum approximation

$$
p_{N}(\tau) \approx P_{N}(\tau)=\langle\delta(N-\mathcal{N}) \exp (\tilde{n}(\mathbf{0}, 0))\rangle
$$

where we have defined

$$
\begin{aligned}
\mathcal{N} & :=\int_{0}^{\infty} \mathrm{d} t \dot{\mathcal{N}}(t), \\
\dot{\mathcal{N}}(t) & :=\int \mathrm{d}^{d} x n(\mathbf{x}, t) .
\end{aligned}
$$

The average in equation (4.1) is performed with the response functional, equation (3.6). One expects the asymptotic scaling behavior $P_{N}(\tau)=N^{-x} f\left(\tau N^{y}\right)$ [1]. In many cases, e.g. absorptive noise as in DP near the critical point, and $N \gg 1$, it is possible to expand the exponential $\exp (\tilde{n}(\mathbf{0}, 0))$ in equation (4.1) in a power series to get asymptotic results. Equation (3.10) leads to $\langle F[n]\rangle=F[0]$ for any functional $F$ of $n$. Hence, the 
first expansion term of the exponential, the 1 , inserted into (4.1) in place of $\exp \tilde{n}(\mathbf{0}, 0)$ leads exactly to $\delta(N)=0$ if $N \gg 1$. The higher powers of $\tilde{n}(\mathbf{0}, 0)$ contribute nonleading corrections to the asymptotic scaling behavior. Hence, only the term $\tilde{n}(\mathbf{0}, 0)$ of the expansion must be retained.

We define a generating function

$$
F(\tau, k)=\sum_{N=0}^{\infty} p_{N}(\tau) \mathrm{e}^{-k N}, \quad k>0 .
$$

This function has the property

$$
F(\tau, k \rightarrow+0)=\left\{\begin{array}{cll}
1 & \text { for } & \tau \geq 0 \\
1-P_{\infty} & \text { for } & \tau<0 .
\end{array}\right.
$$

From the continuous counterpart of (4.3) we obtain, neglecting possible analytic corrections that arise from the small clusters

$$
\begin{aligned}
& F(\tau, k)-F(|\tau|, k)=\int_{0}^{\infty} \mathrm{d} N\left(P_{N}(\tau)-P_{N}(|\tau|)\right) \mathrm{e}^{-k N} \\
& \quad=\langle\exp (\tilde{n}(\mathbf{0}, 0)-k \mathcal{N})\rangle(\tau)-\langle\exp (\tilde{n}(\mathbf{0}, 0)-k \mathcal{N})\rangle(|\tau|) .
\end{aligned}
$$

Here we rely on the property that divergencies like $P_{N} \sim N^{-x}$ for small $N$ are independent of $\tau$ and cancelled in the integrand of equation (4.5) [1. Using now the fact that in the subcritical absorbing phase with unbroken symmetries $\lim _{k \rightarrow+0}\left\langle\mathrm{e}^{-k \mathcal{N}} \exp \tilde{n}(\mathbf{0}, 0)\right\rangle(\tau>0)=\langle\exp \tilde{n}(\mathbf{0}, 0)\rangle(\tau>0)=1$, we arrive at

$$
P_{\infty}=1-\lim _{k \rightarrow+0}\langle\exp (\tilde{n}(\mathbf{0}, 0)-k \mathcal{N})\rangle .
$$

Provided that the asymptotic expansion of the exponential is allowed we get

$$
P_{\infty}=-\lim _{k \rightarrow+0}\left\langle\mathrm{e}^{-k \mathcal{N}} \tilde{n}(\mathbf{0}, 0)\right\rangle \propto|\tau|^{\tilde{\beta}}
$$

by applying equation (3.13). With the help of the duality transformation (3.12) in conjunction with the time translation invariance, we obtain finally

$$
P_{\infty}=\alpha^{2} \lim _{h \rightarrow+0}\left\langle n(\mathbf{0}, \infty) \mathrm{e}^{\lambda h \widetilde{\mathcal{N}}}\right\rangle^{(\text {dual })}=\alpha^{2} \hat{\rho}_{s t} \propto|\tau|^{\hat{\beta}}
$$

where $\tilde{\mathcal{N}}=\int \mathrm{d} t \int \mathrm{d}^{d} x \tilde{n}(\mathbf{x}, t)$ and $\lambda h=k / \alpha^{2}$ constitutes a constant particle source that is taken to zero at the end of the calculation. Note that the particle density of the dual process $\hat{\rho}_{s t}$ is independent of any initial state unequal to the vacuum. In self-dual theories like DP, $\hat{\beta}=\beta$ and the parameter $\alpha$ can be chosen that $\hat{\rho}_{s t}=\rho_{s t}$, as shown above. Then, we see that the redundant variable $\alpha$ enters the relation between the two order parameters: $P_{\infty}=\alpha^{2} \rho_{s t}$

Now we turn our attention to time dependent observables. As above, there are two fundamental complementary quantities, namely the particle density $\rho(t)$ starting from a fully occupied initial state at time $t=0$, and the survival probability $P(t)$ that a cluster grown from a single seed is still active at time $t$. Both are monotonically decreasing functions of time. The exact relation between the two is found with the help of the following consideration. 
Let $p_{N}(t)$ denote the probability that a cluster has exactly $N$ active particles at time $t$. We define the function

$$
P(k, t)=\sum_{N=0}^{\infty}\left(1-\mathrm{e}^{-k N}\right) p_{N}(t) .
$$

Of course, we have $\lim _{k \rightarrow \infty} P(k, t)=P(t)$. We distinguish again between small and large clusters. The former lead to uncritical corrections. For the large clusters we use the approximation

$$
p_{N}(t) \approx P_{N}(t)=\langle\delta(N-\dot{\mathcal{N}}(t)) \exp \tilde{n}(\mathbf{0}, 0)\rangle
$$

where $\dot{\mathcal{N}}(t)$ is defined in equation (4.2b). Thus, we get from the continuous counterpart of equation (4.9)

$$
P(k, t)=\int_{0}^{\infty} \mathrm{d} N\left(1-\mathrm{e}^{-k N}\right) P_{N}(t)=\langle\exp \tilde{n}(\mathbf{0}, 0)\rangle-\langle\exp (\tilde{n}(\mathbf{0}, 0)-k \dot{\mathcal{N}}(t))\rangle .
$$

The first term of the last equation, $\langle\exp \tilde{n}(\mathbf{0}, 0)\rangle$, is equal to one. Hence, we obtain the survival probability

$$
P(t)=1-\lim _{k \rightarrow \infty}\langle\exp (\tilde{n}(\mathbf{0}, 0)-k \dot{\mathcal{N}}(t))\rangle .
$$

After expanding the exponential to get asymptotic results, and utilizing the time translation invariance of the process we arrive at

$$
P(t)=-\lim _{k \rightarrow \infty}\left\langle\tilde{n}(\mathbf{0},-t) \mathrm{e}^{-k \dot{N}(0)}\right\rangle \propto t^{-\tilde{\beta} / \nu z} .
$$

Using again the duality transformation (3.12), we find

$$
P(t)=\alpha^{2} \lim _{\hat{\rho}_{0} \rightarrow \infty}\left\langle n(\mathbf{0}, t) \mathrm{e}^{\hat{\rho}_{0} \dot{\tilde{\mathcal{N}}}(0)}\right\rangle^{(\text {dual })}=\alpha^{2} \hat{\rho}(t) \propto t^{-\hat{\beta} / \hat{\nu} \hat{z}},
$$

where $\dot{\tilde{\mathcal{N}}}(0)=\int \mathrm{d}^{d} x \tilde{n}(\mathbf{x}, 0)$, and $\hat{\rho}_{0}=k / \alpha^{2}$ constitutes an initial constant particle density. Hence, $\hat{\rho}(t)$ is the particle density of the dual process at time $t$ starting from a infinite homogeneous initial density $\hat{\rho}_{0}$, corresponding to a fully occupied initial state. For self-dual processes like DP we have in fact $\hat{\rho}(t)=\rho(t)$ and $\hat{\beta} / \hat{\nu} \hat{z}=\beta / \nu z$. Relation (4.14) is fully analogous to the steady state relation (4.8), but note the different limits $h \rightarrow 0$ and $\hat{\rho}_{0} \rightarrow \infty$. In any case it follows from $\lim _{t \rightarrow \infty} \hat{\rho}(t)=\hat{\rho}_{s t}$ that

$$
\lim _{t \rightarrow \infty} P(t)=P_{\infty} \text {. }
$$

The expressions (4.6, 4.7, 4.12, 4.13) and the relations (4.8, 4.14) are the main general results of this paper. They hold near criticality and for not too small times $t$, because we have neglected several analytic corrections and used, for discrete processes, the continuum approximation. Also we have neglected in (4.7, 4.13) higher powers of $\tilde{n}$. Being interested in non-asymptotic results one has to use the full expression $(\exp \tilde{n}-1)$ instead of the single term $\tilde{n}$ (see (4.6) and (4.12)). 


\section{Probabilities in a mean field approximation}

To demonstrate the applicability of our results (4.7.4.13), we calculate the corresponding path-integrals in a mean-field approximation. Then we are able to write down explicit simple expressions for all quantities and relationships. At first we determine the asymptotic expression of the cluster probability (4.1). Instead of directly evaluating this quantity we consider the Laplace transform

$$
\begin{gathered}
\tilde{M}(k, \tau)=\int_{0}^{\infty} \mathrm{d} N \mathrm{e}^{-k N} P_{N}(\tau)=\langle\exp (\tilde{n}(\mathbf{0}, 0)-k \mathcal{N})\rangle \\
=\int \mathcal{D}(\tilde{n}, n) \exp (-\mathcal{J}[\tilde{n}, n]-(k, n)+\tilde{n}(\mathbf{0}, 0))
\end{gathered}
$$

The saddle point of the exponential weight follows from the solutions of the variational equations

$$
\begin{aligned}
& \frac{\delta(\mathcal{J}[\tilde{n}, n]+(k, n))}{\delta n(\mathbf{x}, t)}=-\frac{\partial \tilde{n}}{\partial t}+\lambda\left(\tau-\nabla^{2}\right) \tilde{n}+\lambda g^{\prime} n \tilde{n}-\frac{\lambda g^{\prime \prime}}{2} \tilde{n}^{2}+k=0, \\
& \frac{\delta(\mathcal{J}[\tilde{n}, n]+(k, n))}{\delta \tilde{n}(\mathbf{x}, t)}=\frac{\partial n}{\partial t}+\lambda\left(\tau-\nabla^{2}\right) n+\frac{\lambda g^{\prime}}{2} n^{2}-\lambda g^{\prime \prime} n \tilde{n}=0 .
\end{aligned}
$$

Replacing $k$ by $k \theta(T-t)$ where the limit $T \rightarrow \infty$ is performed at the end, the solutions of the saddle point equations, subject to the constraints (3.10), are

$$
\begin{aligned}
& \left.\tilde{n}(\mathbf{x}, t)\right|_{s . p .}=\frac{-2 \theta(T-t)(\exp (\lambda w(T-t))-1) k}{\lambda(w+\tau) \exp (\lambda w(T-t))+(w-\tau)}, \\
& \left.n(\mathbf{x}, t)\right|_{s . p .}=0
\end{aligned}
$$

where $w=\sqrt{\tau^{2}+2 g^{\prime \prime} k / \lambda}$. Consequently we have $\left.(\mathcal{J}[\tilde{n}, n]+(k, n))\right|_{\text {s.p. }}=0$, and we obtain in the limit $T \rightarrow \infty$

$$
\tilde{M}(k, \tau)=\left.\exp \tilde{n}(\mathbf{0}, 0)\right|_{s . p .}=\exp \left(\left(\tau-\sqrt{\tau^{2}+2 g^{\prime \prime} k / \lambda}\right) / g^{\prime \prime}\right) .
$$

Using equation (4.6), the limit $k \rightarrow+0$ of this expression yields the percolation probability

$$
P_{\infty}(\tau)=1-\lim _{k \rightarrow+0} \tilde{M}(k, \tau)=1-\exp \left((\tau-|\tau|) / g^{\prime \prime}\right) \approx \frac{2|\tau|}{g^{\prime \prime}} \theta(-\tau) .
$$

The last expression is in accordance with equation (4.8), since $\rho_{s t}(\tau)=2|\tau| \theta(-\tau) / g^{\prime}$ is the stationary solution of the mean field equation.

From equation (5.1) we find the cluster probability $P_{N}$ by an inverse Laplace transform of $\tilde{M}(k, \tau)$

$$
\begin{aligned}
P_{N}(\tau)= & \frac{1}{2 \pi i} \int_{\sigma-i \infty}^{\sigma+i \infty} \mathrm{d} k \mathrm{e}^{k N} \tilde{M}(k, \tau)=\frac{\mathrm{e}^{\tau / g^{\prime \prime}} N^{-3 / 2}}{\sqrt{2 \pi \lambda g^{\prime \prime}}} \exp \left(-\frac{1}{2 g^{\prime \prime}}\left(\lambda \tau^{2} N+(\lambda N)^{-1}\right)\right) \\
& \approx \frac{N^{-3 / 2}}{\sqrt{2 \pi \lambda g^{\prime \prime}}} \exp \left(\tau / g^{\prime \prime}-\lambda \tau^{2} N / 2 g^{\prime \prime}\right)
\end{aligned}
$$

The last row presents indeed the well known asymptotic probability distribution for the generation of a directed $N$-cluster on the Bethe-lattice [1]. $P_{N}(\tau)$ shows the symmetry property

$$
P_{N}(-|\tau|)=\left(1-P_{\infty}(-|\tau|)\right) P_{N}(|\tau|)
$$


between the probability distributions above and below the percolation point with $\int_{0}^{\infty} \mathrm{d} N P_{N}(|\tau|)=1$.

Now we turn our attention to the survival probability (4.12). We have to solve the saddle-point equations (5.2a) where $k$ is replaced by $k \delta(t)$ or, in other words, with the final-condition $\tilde{n}(\mathbf{x}, 0)=-k$ and $k=0$ for $t \neq 0$. We find

$$
\begin{aligned}
& \left.\tilde{n}(\mathbf{x},-t)\right|_{\text {s.p. }}=\frac{-2 \tau \theta(t) \exp (-\lambda \tau t)}{2 \tau k^{-1}+g^{\prime \prime}(1-\exp (-\lambda \tau t))}, \\
& \left.n(\mathbf{x},-t)\right|_{\text {s.p. }}=0 .
\end{aligned}
$$

Hence, according to equation (4.12), the survival probability in mean field approximation is given by

$$
\begin{aligned}
P(t)=1- & \exp \left(\frac{-2 \tau / g^{\prime \prime}}{\exp (\lambda \tau t)-1}\right) \approx \frac{2 \tau / g^{\prime \prime}}{\exp (\lambda \tau t)-1} \\
= & \begin{cases}2 /\left(\lambda g^{\prime \prime} t\right) & \text { for } \tau=0, \\
\left(2 \tau / g^{\prime \prime}\right) \exp (-\lambda \tau t) & \text { for } \tau>0, t \rightarrow \infty, \\
\left(2|\tau| / g^{\prime \prime}\right)(1+\exp (-\lambda|\tau| t)) & \text { for } \quad \tau<0, t \rightarrow \infty .\end{cases}
\end{aligned}
$$

We see that $P(t)$ owns all the required properties displayed in equations (4.14, 4.14).

According to equation (4.11) the inverse Laplace transform of

$$
1-P(k, t)=\langle\exp (\tilde{n}(\mathbf{0},-t)-k \dot{\mathcal{N}}(0))\rangle=\left.\exp \tilde{n}(\mathbf{0},-t)\right|_{s . p}
$$

leads back to

$$
\begin{aligned}
P_{N}(t)=\langle & \delta(N-\dot{\mathcal{N}}(t)) \exp \tilde{n}(\mathbf{0}, 0)\rangle=\frac{1}{2 \pi i} \int_{\sigma-i \infty}^{\sigma+i \infty} \mathrm{d} k(1-P(k, t)) \mathrm{e}^{k N} \\
& =P_{0}(t) \delta(N)+\frac{1}{2 \pi i} \int_{\sigma-i \infty}^{\sigma+i \infty} \mathrm{d} k(P(\infty, t)-P(k, t)) \mathrm{e}^{k N} \\
& =: P_{0}(t) \delta(N)+\bar{P}_{N}(t) .
\end{aligned}
$$

$P_{0}(t)=1-P(t)$ is the extinction probability, and we have used equation (4.12). The subtraction in the integral that defines $\bar{P}_{N}(t)$ is needed for the application of Jordan's lemma. Only after this subtraction the inverse Laplace transform can be calculated by closing the integration path with a large semi-circle which tends to infinity in the left complex $k$-plane. By this subtraction-procedure the $\delta$-contribution of the absorbing state is splitted off. Using the saddle point solution (5.8a) we find

$$
\begin{aligned}
& P_{0}(t)=\exp (-A(t) \bar{N}(t)) \\
& \bar{P}_{N}(t)=A(t) \sqrt{\bar{N}(t) / N} I_{1}(2 A(t) \sqrt{\bar{N}(t) N}) \exp [-A(t)(\bar{N}(t)+N)] .
\end{aligned}
$$

Here we have defined

$$
\begin{aligned}
A(t) & =\frac{2 \tau / g^{\prime \prime}}{1-\exp (-\lambda \tau t)}, \\
\bar{N}(t) & =\exp (-\lambda \tau t) .
\end{aligned}
$$

Note that with the identifications $\lambda=1$ and $g^{\prime \prime}=2$ the mean field solution (15.12a) turns into the distribution calculated with the Fokker-Planck equation (2.4a) with $x_{0}=1$ as we have anticipated in section II. 
Asymptotically, if $A(t) \bar{N}(t) \ll 1$ and $A(t) N$ is finite, we can expand equations (5.12a) and obtain

$$
P_{N}(t) \approx(1-A(t) \bar{N}(t)) \delta(N)+A(t)^{2} \bar{N}(t) \exp (-A(t) N) .
$$

This expression can also be derived if $\exp \tilde{n}(\mathbf{0}, 0)$ is approximated by $1+\tilde{n}(\mathbf{0}, 0)$ in equation (5.11).

For logarithmic corrections to the mean field results in the upper critical dimension $d_{c}=4$ see 31 .

\section{Multiplicative noise and comparison with other work}

Now we equip the Langevin equation (3.1) with multiplicative noise

$$
\overline{\zeta(\mathbf{x}, t) \zeta\left(\mathbf{x}^{\prime}, t^{\prime}\right)}=\lambda g^{\prime \prime} n(\mathbf{x}, t)^{2} \delta\left(\mathbf{x}-\mathbf{x}^{\prime}\right) \delta\left(t-t^{\prime}\right)
$$

instead of the absorptive one, equation (3.2). It is well known that in this case the Langevin equation (3.1) cannot represent a physical reaction-diffusion system [29, 14]. At least diffusional noise must be included and the response functional changes to

$$
\mathcal{J}^{\prime}[\tilde{n}, n]=\int \mathrm{d} t \mathrm{~d}^{d} x\left\{\lambda \tilde{n}\left(\lambda^{-1} \frac{\partial}{\partial t}+\left(\tau-\nabla^{2}\right)+\frac{g^{\prime}}{2} n-\frac{g^{\prime \prime}}{2} \tilde{n} n\right) n-\lambda n(\nabla \tilde{n})\right\} .
$$

The upper critical dimension $d_{c}$ decreases from 4 to 2 . The naive scaling dimensions of the fields become $d$ for $n$ and 0 for $\tilde{n}$. Hence, one expects that also non-Gaussian noise contributions becomes relevant. Indeed the field theory based on $\mathcal{J}^{\prime}$, equation (6.2), is not renormalizable as it stands. However, a quasicanonical transformation to "bosonic" fields

$$
\begin{aligned}
& a^{+}=1+\tilde{a}=\exp \tilde{n}, \\
& a=n \exp (-\tilde{n}),
\end{aligned}
$$

leads to the elimination of the diffusional noise term. The resulting action is known from the theory of branching and annihilating random walks [34. The corresponding Langevin equation is formally equipped with imaginary multiplicative noise.

It is instructive to apply the saddle point approximation to the response functional (6.2). This procedure is expected to be correct above the upper critical dimension. The appropriate saddle point equations are given by (5.2a with the replacement of $g^{\prime \prime}$ by $g^{\prime \prime} n$, and an additional contribution arising from the diffusional noise. However, both terms are cancelled for homogeneous solutions with $n=0$. We get the time-dependent solution

$$
\left.\tilde{n}(\mathbf{x},-t)\right|_{s . p .}=-k \exp (-\lambda \tau t) .
$$

which goes to infinity for $k \rightarrow \infty$. Thus, for each finite time $t$ we obtain from equation (4.12)

$$
P(t)=1-\left.\lim _{k \rightarrow \infty} \exp \tilde{n}(\mathbf{x},-t)\right|_{s . p .}=1 .
$$

Hence, the survival probability for processes equipped with multiplicative behaves trivially at least in the saddle point approximation. It is obvious that an expansion 
of $\exp \tilde{n}$ is not allowed. Indeed, it is appropriate to change to the bosonic variables (6.3a).

Now we are in a position to compare our result for the survival probability with the corresponding expression given by MGT. As we have seen, the distribution function $P_{N}(t)$ (5.11) consists of two parts

$$
\begin{aligned}
P_{N}(t) & =P_{N}(t)=\langle\delta(N-\dot{\mathcal{N}}(t)) \exp \tilde{n}(\mathbf{0}, 0)\rangle \\
& =(1-P(t)) \delta(N)+P(t) \bar{p}_{N}(t)
\end{aligned}
$$

where the nonsingular distribution $\bar{p}_{N}(t)$ is normalized to $\int_{0}^{\infty} \mathrm{d} N \bar{p}_{N}(t)=1$. The survival probability $P(t)$ is a monotonic decreasing function in the case of absorptive noise and equal to one in the case of multiplicative one. MGT have introduced the expression

$$
P^{(M G T)}(t, \alpha)=\langle\theta(\dot{\mathcal{N}}(t)-\alpha) \tilde{n}(\mathbf{0}, 0)\rangle
$$

as a substitute of the survival probability. In the case of absorptive noise, where asymptotically the expansion of $\exp \tilde{n}$ is allowed, we get from equation (6.6)

$$
\langle\delta(N-\dot{\mathcal{N}}(t)) \tilde{n}(\mathbf{0}, 0)\rangle=P(t)\left[\bar{p}_{N}(t)-\delta(N)\right] .
$$

Hence, one sees immediately

$$
\lim _{\alpha \rightarrow 0} P^{(M G T)}(t, \alpha)=P(t)
$$

in the large $t$ and small $\tau$ limit. The advantage of our expression (4.13) for the survival probability is its calculability in perturbation theory. This contrasts to the substitute (6.7), in which the Heaviside- $\theta$-function is a insurmountable complication for any calculation.

Things become worse for multiplicative noise. As we have shown, the expansion of $\exp \tilde{n}$ is dubious, and the survival probability $P(t)=1$ as long as $t$ is finite. Even if the distribution $\bar{p}_{N}(t)$ piles up near $N=0$ with $\bar{p}_{N}(t) \sim N^{\rho}$ for small $N$ and a width $B(t)$, the substitute behaves like

$$
P^{(M G T)}(t, \alpha)=1+O\left((\alpha / B(t))^{\rho+1}\right)
$$

and goes to zero exponentially if $\alpha \gg B(t)$. Hence, $\alpha$ is a dangerous variable, and it is not clear which value should be assigned to it.

\section{Other growth models with absorptive noise}

In this section we will briefly discuss the applicability of our expressions to other growth processes with absorbing states widely discussed in the literature. We present several variants of the original DP, for which the expressions (4.74.13) for the probabilities are well applicable. 


\subsection{Tricritical directed percolation}

Tricritical directed percolation (TDP) can be understood as the limit of DP in which the renormalized coupling constant $g^{\prime}$ goes to zero. In this limit no growth limiting term shows up in the original mean field part of the equation of motion of DP (3.13.3). We expect that formerly irrelevant couplings become now relevant. The nature of these terms depends on the physics of the growth process under consideration. The simplest case is the occurrence of a higher order effective coupling in the reaction rate. In TDP 35, 36] one assumes the form

$$
R[n]=\lambda\left(\tau+\frac{g^{\prime}}{2} n+\frac{f}{6} n^{2}\right)
$$

that leads to an interaction part of the response functional (3.6)

$$
\mathcal{V}_{T D P}[\tilde{n}, n]=\frac{1}{2}\left(g^{\prime} n+\frac{f}{3} n^{2}-g^{\prime \prime} \tilde{n}\right) .
$$

In the mean field approximation, the tricritical point which separates the continuous DP-transition from a discontinuous one is found at $\tau=g^{\prime}=0$. The noise term has the absorbing form (3.2). However, the dual symmetry (3.12) is lost and we expect different exponents $\beta \neq \tilde{\beta}=\beta^{\prime}$. Indeed, a field-theoretic renormalization group calculation up to 2-loop order in an $\varepsilon$-expansion below the upper critical dimension $d_{c}=3, \varepsilon=3-d$, yields

$$
\beta=\frac{1}{2}-0.458 \varepsilon+O\left(\varepsilon^{2}\right), \quad \beta^{\prime}=1+O\left(\varepsilon^{2}\right) .
$$

The other exponents are $z=2+0.0086 \varepsilon+O\left(\varepsilon^{2}\right)$ and $\nu=1 / 2+0.0075 \varepsilon+O\left(\varepsilon^{2}\right)$. The crossover exponent $\phi=1 / 2-0.012 \varepsilon+O\left(\varepsilon^{2}\right)$ describes the scaling of the crossover from TDP to DP. It can be easily seen that the mean-field calculations of the probabilities in section $\mathrm{V}$ lead to the same results for TDP as found for DP.

\subsection{Compact directed percolation}

The universality class of compact directed percolation (CDP) that includes e.g. the voter model 6, 37, 38, 39, describes another universality class that can be reached in the limit

$g^{\prime} \rightarrow 0$ of DP. In contrast to TDP, in CDP a limiting term in the deterministic part of the continuum Langevin equation of motion (3.13.3) does not exist a priori. Thus, in the active phase with $\tau<0$, the density grows to infinity corresponding to a state with a completely filled lattice. Renormalizing the density of the filled lattice to the value $n=1$, we expect that formerly irrelevant couplings even in the noise become relevant. A special physical condition comes from the demand that the empty and the filled lattice are both absorbing states and the exchange $n \rightarrow 1-n, \tau \rightarrow-\tau$ is a symmetry. The patchy spatial structure of the CDP-states motivates an expansion of the equation of motion in the small quantity $n(1-n)$. Hence, the relevant terms of the reaction rate of the Langevin equation (3.13.3) are given by

$$
R[n]_{(\mathbf{x}, t)} n(\mathbf{x}, t)=\lambda \tau(1-n(\mathbf{x}, t)) n(\mathbf{x}, t) .
$$


The reaction rate is zero at the critical point $\tau=0$. For $\tau \neq 0$, the model has a bias which prefers the state $n=0$ or $n=1$ depending on the sign of $\tau$. The noise in the empty or the filled lattice is zero. This enforces Gaussian fluctuations of the noise of the form 39]

$$
\overline{\zeta(\mathbf{x}, t) \zeta\left(\mathbf{x}^{\prime}, t^{\prime}\right)}=\lambda g n(\mathbf{x}, t)(1-n(\mathbf{x}, t)) \delta\left(\mathbf{x}-\mathbf{x}^{\prime}\right) \delta\left(t-t^{\prime}\right) .
$$

equations (7.47.5) lead to the response functional (3.6) of CDP

$$
\mathcal{J}_{C D P}[\tilde{n}, n]=\int \mathrm{d} t d^{d} x\left\{\lambda \tilde{n}\left(\lambda^{-1} \frac{\partial n}{\partial t}-\nabla^{2} n+\tau n(1-n)-\frac{g}{2} \tilde{n} n(1-n)\right)\right\}
$$

This response functional is invariant under the transformation $n \rightarrow 1-n, \tilde{n} \rightarrow-\tilde{n}$, $\tau \rightarrow-\tau$. For $\tau=0, \mathcal{J}_{C D P}[\tilde{n}, n]$ transforms under duality (3.12) to an action which describes annihilating random walks [40, 41]. Note that the noise then becomes imaginary in the corresponding Langevin equation. Physically this results from the anticorrelations present in the annihilating walk process. Consequently, the notion of density variables are inappropriate in such a case. Instead the fields $n, \tilde{n}$ represent annihilation and creation operators of a bosonic field theory. The scaling dimensions of $\tilde{n}$ and $n$ are not related, and are given trivially by the naive dimensions which are $d$ and 0 , respectively. The upper critical dimension is $d_{c}=2$. The renormalization of the bias $\tau$ is intimately related to the exactly computable renormalization of the coupling constant $g$. A short presentation of the field theory is given in the appendix.

We note here the well known order parameter exponents of CDP for $d \leq 2$

$$
\beta=0, \quad \beta^{\prime}=1 .
$$

The dynamical and the correlation length exponents are $z=2$ and $\nu=1 / d$. The crossover exponent to DP is found to be $\phi=2 / d$.

For $d \geq 2$, the mean-field results for the probabilities derived for DP are applicable. The survival probability for clusters generated from a localized seed in an otherwise empty lattice at and below $d=2$, as well as the percolation probability follows from our general formulas (4.7) and (4.13) to

$$
P(t)=t^{-d / 2} f\left(\tau t^{d / 2}\right), \quad P_{\infty} \propto|\tau| .
$$

\subsection{Conserved diffusing secondary field}

In analogy to the extension of Model A to Model C from the Halperin-Hohenberg catalog of fundamental field-theoretic models for critical dynamics, one can consider an extension of DP to DP-C [43]. In Model C a conserved diffusing density which is not critical itself is coupled to the relaxing dynamics of an order parameter. In the DP-C universality class, a conserved density $c(\mathbf{x}, t)$ is coupled to the growing density field $n(\mathbf{x}, t)$ of the DP-process. The relevant Langevin dynamics is given by

$$
\begin{aligned}
& \partial_{t} n=\lambda \nabla^{2} n-\lambda\left(\tau+\frac{g^{\prime}}{2} n+f c\right) n+\zeta, \\
& \partial_{t} c=\nabla^{2}(\gamma c+\sigma n)+\zeta^{\prime}
\end{aligned}
$$


where the Langevin forces are Gaussian absorptive noise (3.2) and Gaussian diffusion noise

$$
\overline{\zeta^{\prime}(\mathbf{x}, t) \zeta^{\prime}\left(\mathbf{x}^{\prime}, t^{\prime}\right)}=-\gamma \nabla^{2} \delta\left(\mathbf{x}-\mathbf{x}^{\prime}\right) \delta\left(t-t^{\prime}\right) .
$$

The first model of this type (with $\sigma=0$ ) was introduced by Kree et al. 44] to deal with a catalytic poison which may damage a growing population. Ten years later, van Wijland et al. [45] have shown that a special reaction-diffusion system $(A+B \rightarrow 2 B, B \rightarrow A)$ corresponds in general to DP-C with a cross-diffusion coefficient $\sigma \neq 0$. However, if $\sigma f>0$, the renormalization flow leads finally to a violation of the stability bound $2 \sigma f<g$ of the mean field part of equations (17.9a), which is interpreted as the occurrence of a fluctuation induced first order transition. In this sense, the KSS model $(\sigma=0)$ defines a tricritical point of the critical DP-C model with $\sigma f<0$ and with its own universality class. Note that the limit $\gamma \rightarrow 0$ leads to another universality class [46] called the conserved lattice gas (CLG) or Manna class [47. The systems belonging to this universality class exhibit infinitely many absorbing states characterized by the different distributions of a conserved quantity. A recent renormalization group approach [48 is dubious. It proposes an upper critical dimension $d_{c}=6$, whereas simulations [49, 50] clearly exhibit an upper critical dimension $d_{c}=4$.

As the result of special symmetries, the DP-C model has the exact exponents $z=2$ and $\nu=2 / d$ below the upper critical dimension $d_{c}=4$. The special KSS model is invariant under the duality transformation (3.12) extended by $c(\mathbf{x}, t) \rightarrow c(\mathbf{x},-t)$. Hence, for $\sigma=0$ we have the equality

$$
\beta=\beta^{\prime}=1-\frac{\varepsilon}{16}+O\left(\varepsilon^{2}\right)
$$

where the expansion results from a 1-loop calculation [44. In general, for $\sigma \neq 0$, dual symmetry is lost. In this case it turns out, that $n$ does not renormalize. Its anomalous dimension is zero whereas the anomalous dimension of $\tilde{n}$ is nontrivial. One obtains 45] finally

$$
\beta=1, \quad \beta^{\prime}=1-0.1065 \varepsilon+O\left(\varepsilon^{2}\right) .
$$

Also for the DP-C as well as the CLG class, the mean field calculation of the survival and percolation probability lead to the formulas known from DP and is expected to be correct above the upper critical dimension.

\subsection{Dynamic isotropic percolation}

Contrary to the growth models considered up to now, the dynamic isotropic percolation (dIP) modelled by the general epidemic process (GEP) [51] leads to memory terms in the equation of motion for the growing density. The debris (or the immunes in the language of the GEP) is generated by the agent, i.e. the active particles (or the infected individuals). It suppresses the growth process itself and leads finally to the extinction of the disease ( $n=0$ in each finite spatial region). The spatial distribution of the debris in the stationary limit is described by the usual static percolation statistics. Thus, it is 
more appropriate to consider the density of the debris as an order parameter field. It is proportional to the time integral of the density of the active particles

$$
m(\mathbf{x}, t)=\lambda \int_{-\infty}^{t} \mathrm{~d} t^{\prime} n\left(\mathbf{x}, t^{\prime}\right) .
$$

The relevant terms in the reaction rate of the Langevin equation (3.1) are now given by

$$
R[n]_{(\mathbf{x}, t)}=\lambda\left(\tau+g^{\prime} m(\mathbf{x}, t)\right) .
$$

Therefore, the response functional is follows as [27]

$$
\mathcal{J}_{d I P}[\tilde{n}, n]=\int \mathrm{d} t \mathrm{~d}^{d} x\left\{\lambda \tilde{n}\left(\lambda^{-1} \frac{\partial}{\partial t}+\left(\tau-\nabla^{2}\right)+g^{\prime} m-\frac{g^{\prime \prime}}{2} \tilde{n}\right) n\right\} .
$$

dIP is not invariant under the transformation (3.12). However, the variant of the duality transformation

$$
\alpha^{-1} \tilde{n}(\mathbf{x}, t) \rightarrow-\alpha m(\mathbf{x},-t)
$$

with $\alpha=\sqrt{g^{\prime} / g^{\prime \prime}}$ transforms the response functional (7.15) onto itself. This shows that the particle density of the dual dynamic percolation can be identified with the field of the debris of the primal dynamic percolation process. Relation (7.16) leads to the identity $\beta-\nu z=\tilde{\beta}$ between the exponents defined in (3.13), and shows that one has to identify $\tilde{\beta}=\beta_{P}$, where $\beta_{P}$ is the order parameter exponent defined in static percolation theory. It is easy to see that the mean field result (5.9) for the survival probability is applicable above six spatial dimensions also in the case of dynamic isotropic percolation. For logarithmic corrections in the upper critical dimension $d_{c}=6$ see [52]. Whereas the formulas (4.7, 4.13) are correct in general, one now has

$$
P_{\infty}=\alpha^{2} \lim _{h \rightarrow+0}\left\langle m(\mathbf{0}, \infty) \mathrm{e}^{\lambda h \dot{\mathcal{N}}(0)}\right\rangle=\alpha^{2} \rho_{s t} \propto|\tau|^{\tilde{\beta}} .
$$

instead of equation (4.8) as a consequence of the symmetry (17.16). Here $\rho_{s t}$ is the stationary homogeneous density of the debris in the active percolating phase.

\subsection{Tricritical dynamic isotropic percolation}

As a last example we consider briefly the tricritical variant of dynamic isotropic percolation (TdIP) [53, 54]. TdIP as a tricritical point followed by a first order transition can be founded on a generalization of the GEP. One introduces in the reaction scheme of the GEP weakened individuals as a further species. This species is weakened by an infected neighbor but not immune. Hence, a second infection in its neighborhood makes this weakened individuals more easy susceptible. In the consequence, this property introduces an instability that may lead to a discontinuous epidemic transition. The response functional (7.15) is extended to

$$
\mathcal{J}_{\text {TdIP }}[\tilde{n}, n]=\int \mathrm{d} t \mathrm{~d}^{d} x\left\{\lambda \tilde{n}\left(\lambda^{-1} \frac{\partial}{\partial t}+\left(\tau-\nabla^{2}\right)+f m+\frac{g^{\prime}}{2} m^{2}-\frac{g^{\prime \prime}}{2} \tilde{n}\right) n\right\} .
$$


The tricritical point is given in mean field theory by $\tau=f=0$. The upper critical dimension is $d=d_{c}=5$. The symmetry (7.16) is lost. Hence, we find four independent critical exponents. In a $\varepsilon$-expansion with $\varepsilon=5-d$ we obtained

$$
\beta=\frac{3}{2}-\frac{74}{225} \varepsilon+O\left(\varepsilon^{2}\right), \quad \beta^{\prime}=1-\frac{2}{45} \varepsilon+O\left(\varepsilon^{2}\right) .
$$

The mean field result (5.9) for the survival probability is applicable above five spatial dimensions also in this case. For logarithmic corrections in the upper critical dimension $d_{c}=5$ see 54

\section{Epilogue}

Survival and percolation probabilities are most important quantities in the theory and in the application of spreading. In the past, one has missed calculable expressions in the field theory of this phenomena. We have seen that it is possible to derive such expressions, and that their calculations are feasible in perturbation expansions. We have presented the results in a simple mean field approximation. Extension to higher order of perturbation series are possible without difficulties. We have presented results in [31, 52, 54].

The outstanding role of the form of the absorptive noise is stressed. So called multiplicative noise would lead to trivial non-decaying survival probabilities. In the last chapter we have shortly considered some fundamental growth models equipped with absorptive noise that are representations of known universality classes of spreading

phenomena. All considerations are applicable also to models with long-range spreading [55]

\section{Acknowledgments}

I thank Georg Foltin and Olaf Stenull for a critical reading of the manuscript. This work has been supported by the Deutsche Forschungsgemeinschaft via the Sonderforschungsbereich 237 "Unordnung und große Fluktuationen".

\section{Appendix: Renormalized field theory of compact directed percolation}

It is well known that CDP offers many exactly calculable properties. The response functional of the CDP process with bias (77.6) equipped with a further relevant term to describe the crossover to DP is

$$
\begin{aligned}
\mathcal{J}[\tilde{n}, n]= & \int \mathrm{d} t \mathrm{~d}^{d} x\left\{\lambda \tilde{n}\left(\lambda^{-1} \frac{\partial n}{\partial t}-\nabla^{2} n+(\tau+r) n(1-n)+r n^{2}-\frac{g}{2} \tilde{n} n(1-n)\right)\right\} \\
& =\int \mathrm{d} t \mathrm{~d}^{d} x\left\{\lambda \tilde{n}\left(\lambda^{-1} \frac{\partial n}{\partial t}-\nabla^{2} n+r n+\tau n(1-n)-\frac{g}{2} \tilde{n} n(1-n)\right)\right\} .
\end{aligned}
$$

The new coupling term with $r \geq 0$ suppresses now the density of the active particles in the formerly compact filled regions in contrast to the other terms. The deterministic 
term proportional to $\tau$ and the noise proportional to $g$ are effectively present only at the interface between empty and filled regions [42. These interfaces are broken up if $r>0$ and the process crosses over to DP. Note that in a mean field picture the transition line between the absorbing and the active phase is given by $\tau_{c}=-r$.

The renormalized field theory of the critical voter model below $d=d_{c}=2$, that is A.1 with $\tau=r=0$, is usually developed by mapping the model to the problem of annihilating random walks. The mapping consists of two steps. At first a duality transformation is applied, then the diagrams contributing to the relevant vertex functions [40, 41] are summed up exactly. This can be done also directly for the field theory defined by the functional (A.1). Including the parameter $r$ in the propagator $G(t, q, r)=\theta(t) \exp \left(-\lambda\left(q^{2}+r\right) t\right)$, we find for the primitively diverging vertex functions $\Gamma_{\tilde{k}, k}$, where $\tilde{k}$ and $k$ denote the numbers of the amputated $\tilde{n}$ - and $n$-legs of the irreducible diagrams, exactly

$$
\begin{aligned}
& \Gamma_{2,2}=-2 \Gamma_{2,1}=2 \lambda g D \\
& \Gamma_{1,2}=-2 \Gamma_{1,1}+2\left(i \omega+\lambda q^{2}+\lambda r\right)=-2 \lambda \tau D .
\end{aligned}
$$

Here,

$$
D=\frac{1}{1+g I},
$$

is the correction that arises from the summation of all contributing diagrams, the chains of "bubbles". The single bubble integral is given by

$$
\begin{aligned}
I(\omega, q, r) & =\lambda \int_{0}^{\infty} \mathrm{d} t \mathrm{e}^{-i \omega t} \int_{p} G\left(t, \frac{q}{2}+p, r\right) G\left(t, \frac{q}{2}-p, r\right) \\
& =\frac{\Gamma(1+\varepsilon / 2)}{\varepsilon(4 \pi)^{d / 2}} g\left(r+\frac{q^{2}}{4}+\frac{i \omega}{2 \lambda}\right)^{-\varepsilon / 2},
\end{aligned}
$$

where $\varepsilon=2-d$. We renormalize $g$ by $g \rightarrow \stackrel{\circ}{g}=Z g$, and choose the renormalization point RP by $\left(r+q^{2} / 4+i \omega / 2 \lambda\right)=\mu^{2}$, where $\mu$ is the usual convenient inverse length scale. Furthermore, we define

$$
\left.\stackrel{\circ}{g} D\right|_{R P}=\frac{\stackrel{\circ}{g}}{1+\left.\stackrel{\circ}{g} I\right|_{R P}}=: g=A_{\varepsilon} u \mu^{\varepsilon}
$$

with $A_{\varepsilon}=\Gamma(1+\varepsilon / 2) /(4 \pi)^{d / 2}$. We obtain finally the renormalization constant

$$
Z=\frac{1}{1-u / \varepsilon}
$$

Equations (A.2) show that $\tau$ renormalizes in the same way as $g$, whereas $r$ needs no renormalization:

$$
\tau \rightarrow \stackrel{\circ}{\tau}=Z \tau, \quad r \rightarrow \stackrel{\circ}{r}=r
$$

With the help of this procedure all $\varepsilon$-poles are eliminated from the perturbation series.

In the same way one can study the correlation functions with the composite interface field $\varphi=n(1-n)$. An insertion of the field $\varphi$ requires a further renormalization. It can be easily seen that this renormalization is given by $\varphi \rightarrow \stackrel{\circ}{=}=Z^{-1} \varphi$. Thus, all 
renormalizations of the response functional (A.1) follow from the renormalization of this interface field. The cumulant of $k n$-fields, $\tilde{k} \tilde{n}$-fields and $m \varphi$-fields, starting from uncorrelated initial conditions with $n(\mathbf{x}, t=0)=n_{0}$ is denoted by $G_{k, \tilde{k} ; m}$. The renormalization group equation (RGE) for the Greens functions $G_{k, \tilde{k} ; m}$, reads

$$
\left(\mu \partial_{\mu}+\beta \partial_{u}+\kappa \tau \partial_{\tau}+m \kappa\right) G_{k, \tilde{k} ; m}\left(\{\mathbf{x}, t\}, \tau, r, n_{0}, u, \lambda, \mu\right)=0
$$

where the RG-functions result from the logarithmic derivative of the $Z$-factor, $\kappa=$ $-\partial \ln Z /\left.\partial \ln \mu\right|_{0}$, holding bare parameters constant. We get

$$
\kappa=u, \quad \beta=(-\varepsilon+u) u .
$$

At the stable fixed point of the RGE, $u_{*}=\varepsilon>0$, we arrive the scaling form

$$
G_{k, \tilde{k} ; m}\left(\{\mathbf{x}, t\}, \tau, r, n_{0}, u_{*}\right)=l^{\tilde{k} d+m \varepsilon} G_{k, \tilde{k} ; m}\left(\left\{l \mathbf{x}, l^{2} t\right\}, \tau l^{-d}, r l^{-2}, n_{0}, u_{*}\right) .
$$

All the critical exponents follow from these equations. The DP critical line, the line between the absorbing and the active phase for $r>0$, results as $\tau_{c} \propto-r^{d / 2}$. From the asymptotic solution of the RGE directly in two spatial dimensions, one can infer

$$
\begin{gathered}
G_{k, \tilde{k} ; m}\left(\{\mathbf{x}, t\}, \tau, r, n_{0}, u\right)=l^{2 \tilde{k}} X(l)^{m} G_{k, \tilde{k} ; m}\left(\left\{l \mathbf{x}, l^{2} t\right\}, X(l) l^{-2} \tau, r l^{-2}, n_{0}, X(l) u\right), \\
X(l)^{-1}=1-u \ln l .
\end{gathered}
$$

This general scaling relation determines all the logarithmic corrections known for CDP in $d=2$. One gets, e.g., for the mean interface density at the critical point $\tau=0$ and with a non-universal time constant $t_{0}$, asymptotically

$$
\langle\varphi(\mathbf{x}, t)\rangle \propto n_{0}\left(1-n_{0}\right)\left(\ln t / t_{0}\right)^{-1}+O\left(\left(\ln t / t_{0}\right)^{-2}\right),
$$

whereas $\langle n(\mathbf{x}, t)\rangle=n_{0}=$ const, a relation well known from the exact solution of the two-dimensional lattice voter model [56, 57].

[1] D. Stauffer and A. Aharony, Introduction to Percolation Theory (Taylor \& Francis, London, 1994).

[2] A. Bunde, S. Havlin, eds. Fractals in Science, Fractals and Disordered Systems (Springer, Berlin, 1995/96).

[3] H. Hinrichsen, Adv. Phys. 49, 815 (2001).

[4] D. Mollison, J. R. Stat. Soc. B 39, 283 (1977).

[5] N.T.J. Bailey, The Mathematical Theory of Infectious Diseases (Griffin, London, 1985).

[6] T.M. Liggett, Interacting Particle Systems (Springer, New York, 1985).

[7] J.D. Murray, Mathematical Biology (Springer, Berlin, 1989).

[8] H.K. Janssen, Z. Phys. B: Cond. Mat. 23, 377 (1976); R. Bausch, H.K. Janssen, and H. Wagner, ibid. 24, 113 (1976); H.K. Janssen, in: Dynamical Critical Phenomena and Related Topics, Lecture Notes in Physics, Vol. 104, ed. C.P. Enz (Springer, Heidelberg, 1979).

[9] C. DeDominicis, J. Physique C 37, 247 (1976); C. DeDominicis and L. Peliti, Phys. Rev. B 18, 353 (1978).

[10] H.K. Janssen, in: From Phase Transition to Chaos, ed. G. Györgyi, I. Kondor, L. Sasvári, T. Tél (World Scientific, Singapore, 1992).

[11] For a recent review of the field theory approach to percolation processes see H.K. Janssen and U.C. Täuber, to be published in Ann. of Phys. (2005).

[12] M.A. Muñoz, G. Grinstein, and Y. Tu, Phys. Rev. E 56, 5101 (1997).

[13] D.R. Hofstadter, Gödel, Escher, Bach (Basic Books, 1979).

[14] M.A. Muñoz, Phys. Rev. E 57, 1377 (1998). 
[15] W. Feller, Ann. Math. 55, 468 (1952); Commun. Pure Appl. Math. 8, 203 (1955); An Introduction to Probability Theory and its Applications, 2 Vols., (2nd edition, Wiley, New York 1957).

[16] A.T. Bharucha-Reid, Elements of the Theory of Markov Processes and their Application (McGrawHill, New York, 1960).

[17] C.W. Gardiner, Handbook of Stochastic Methods (Springer, Berlin, 1985).

[18] N.G. van Kampen, Stochastic Processes in Physics and Chemistry (2nd edition, North-Holland, Amsterdam 1992).

[19] I.I. Gichman and A.W. Skorochod, Stochastische Differentialgleichungen, (Akademie-Verlag, Berlin 1971).

[20] R. Graham and A. Schenzle, Phys. Rev. A 25, 1731 (1982).

[21] S.R. Broadbent and J.M. Hammersley, Proc. Camb. Philos. Soc. 53, 629 (1957).

[22] P. Grassberger and K. Sundermeyer, Phys. Lett. B 77, 220 (1978); P. Grassberger and A. de la Torre, Ann. Phys. NY 122, 373 (1979).

[23] J.L. Cardy and R.L. Sugar, J. Phys. A: Math. Gen. 13, L423 (1980).

[24] S.P. Obukhov, Physica A 101, 145 (1980).

[25] H.K. Janssen, Z. Phys. B: Cond. Mat. 42, 151 (1981).

[26] W. Kinzel, in: Percolation Structures and Processes, ed. G. Deutscher, R. Zallen, and J. Adler, Annals of the Israel Physical Society, Volume 5 (Adam Hilger, Bristol, 1983); Z. Phys. B: Cond. Mat. 58, 229 (1985).

[27] H.K. Janssen, Z. Phys. B: Cond. Mat. 58, 311 (1985).

[28] H.K. Janssen, Journ. Stat. Phys. 103, 801 (2001).

[29] M.J. Howard and U.C. Täuber, J. Phys. A: Math. Gen. 30, 7721 (1997).

[30] J.F.F. Mendes, R.D. Dickman, M. Henkel, M.C. Marques, J. Phys. A: Math. Gen. 27, 3019 (1994).

[31] H.K. Janssen and O. Stenull, Phys. Rev. E 69, 016125 (2004).

[32] D.J. Amit, Field Theory, the Renormalization Group, and Critical Phenomena (World Scientific, Singapore, 1984).

[33] J. Zinn-Justin, Quantum Field Theory and Critical Phenomena (Clarendon, Oxford, 1996).

[34] J.L. Cardy and U.C. Täuber, Phys. Rev. Lett. 77, 4870 (1996); Journ. Stat. Phys. 90, 1 (1998).

[35] T. Ohtsuki and T. Keyes, Phys. Rev. A 35, 2697 (1987), ibid. A 36, 4434 (1987).

[36] H.K. Janssen, J. Phys. A: Math. Gen. 20, 5733 (1987) and unpublished.

[37] E. Domany and W. Kinzel, Phys. Rev. Lett. 47, 5 (1981); Phys. Rev. Lett. 53, 311 (1984).

[38] J.W.Essam, J. Phys. A: Math. Gen. 22, 4927 (1989).

[39] R. Dickman and A. Yu, Phys. Rev. E 52, 3218 (1995).

[40] L. Peliti, J. Phys. A: Math. Gen. 19, L365 (1986).

[41] B.P. Lee, J. Phys. A: Math. Gen. 27, 2633 (1994); B.P. Lee and J.L. Cardy, Journ. Stat. Phys. 80, 971 (1995).

[42] I. Dornic, H. Chaté, J. Chave, and H. Hinrichsen, Phys. Rev. Lett. 87, 045701 (2001).

[43] H.K. Janssen, Phys. Rev. E 64, 058101 (2001) and unpublished.

[44] R. Kree, B. Schaub, and B. Schmittmann, Phys. Rev. A 39, 2214 (1989).

[45] F. van Wijland, K. Oerding, and H.J. Hilhorst, Physica A 61, 6330 (2000).

[46] M. Rossi, R. Pastor-Satorras, and A. Vespignani, Phys. Rev. Lett. 85, 1803 (2000).

[47] S.S. Manna, J. Phys. A: Math. Gen. 24, L363 (1991).

[48] F. van Wijland, Phys. Rev. Lett. 89, 190602 (2002).

[49] S. Lübeck, Phys. Rev. E 64, 016123 (2001); ibid. 65, 046150 (2002); ibid. 66, 046114 (2002).

[50] S. Lübeck and P.C. Heger, Phys. Rev. Lett. 90, 230601 (2003).

[51] P. Grassberger, Math. Biosci. 63, 157 (1983); J.L. Cardy and P. Grassberger, J. Phys. A: Math. Gen. 18, L267 (1985).

[52] H.K. Janssen and O. Stenull, Phys. Rev. E 68, 036131 (2003).

[53] M. Müller, Diploma-thesis, Heinrich-Heine-Universität Düsseldorf (1994).

[54] H.K. Janssen, M. Müller, and O. Stenull, Phys. Rev. E 70, 026114 (2004).

[55] H.K. Janssen, K. Oerding, F. van Wijland, and H.J. Hilhorst, Eur. Phys. J. B 7, 137 (1999). 
[56] M. Scheucher and H. Spohn, Journ. Stat. Phys. 53, 279 (1988).

[57] P.L. Krapivsky, Phys. Rev. A 45, 1067 (1992); L. Frachebourg and P.L. Krapivsky, Phys. Rev. E 53, R3009 (1996). 